\title{
Exchange-field enhancement of superconducting spin pumping
}

\author{
Kun-Rok Jeon, ${ }^{1,2}$ Chiara Ciccarelli, ${ }^{2, *}$ Hidekazu Kurebayashi, ${ }^{3}$ Lesley F. Cohen, ${ }^{4}$ Xavier Montiel, ${ }^{5}$ Matthias Eschrig, \\ Sachio Komori, ${ }^{1}$ Jason W. A. Robinson, ${ }^{1}$ and Mark G. Blamire ${ }^{1, \dagger}$ \\ ${ }^{1}$ Department of Materials Science and Metallurgy, University of Cambridge, 27 Charles Babbage Road, \\ Cambridge CB3 OFS, United Kingdom \\ ${ }^{2}$ Cavendish Laboratory, University of Cambridge, Cambridge CB3 OHE, United Kingdom \\ ${ }^{3}$ London Centre for Nanotechnology and Department of Electronic and Electrical Engineering at University of College London, \\ London WC1H $01 \mathrm{H}$, United Kingdom \\ ${ }^{4}$ The Blackett Laboratory, Imperial College London, London SW7 2AZ, United Kingdom \\ ${ }^{5}$ Department of Physics, Royal Holloway, University of London, Egham Hill, Egham, Surrey TW20 OEX, United Kingdom
}

(Received 1 November 2018; revised manuscript received 19 December 2018; published 15 January 2019)

\begin{abstract}
A recent ferromagnetic resonance study [Jeon et al., Nat. Mater. 17, 499 (2018)] has reported that spin pumping into a singlet superconductor $(\mathrm{Nb})$ can be greatly enhanced over the normal state when the $\mathrm{Nb}$ is coupled to a large spin-orbit-coupling (SOC) spin sink such as Pt. This behavior has been explained in terms of the generation of spin-polarized triplet supercurrents via SOC at the $\mathrm{Nb} / \mathrm{Pt}$ interface, acting in conjunction with a nonlocally induced magnetic exchange field. Here we report the effect of adding a ferromagnet $(\mathrm{Fe})$ to act as an internal source of an additional exchange field to the adjacent Pt spin sink. This dramatically enhances the spin pumping efficiency in the superconducting state compared with either Pt and Fe separately, demonstrating the critical role of the exchange field in generating superconducting spin currents in the $\mathrm{Nb}$.
\end{abstract}

DOI: 10.1103/PhysRevB.99.024507

Spin-triplet Cooper pairs can carry a nondissipative spin current and are an essential element for the emergent field of superconducting spintronics [1-3]. In the past decade, the generation of spin-polarized triplet pairs within ferromagnets via spin mixing and spin rotation processes at magnetically inhomogeneous superconductor/ferromagnet (SC/FM) interfaces has been intensively studied [1-4] based on the Josephson effect in SC/FM/SC junctions [5] and the critical temperature $T_{\mathrm{c}}$ modulation in $\mathrm{FM} / \mathrm{SC} / \mathrm{FM}$ and $\mathrm{SC} / \mathrm{FM} / \mathrm{FM}^{\prime}$ superconducting spin valves [6,7].

Recent theoretical works $[8,9]$ have suggested spin-orbit coupling (SOC) in combination with a magnetic exchange field $h_{\mathrm{ex}}$ as an alternative mechanism to generate the spinpolarized triplet supercurrents even at a single magnetically homogeneous SC/FM interface. Briefly, in the presence of $h_{\mathrm{ex}}$, some of the spin singlets forming the superconducting condensate of a conventional SC are converted into spin-zero triplets oriented along $h_{\mathrm{ex}}$. If the SOC, originating either from bulk (Dresselhaus-type) or structure (Rashba-type) inversion asymmetry, could have the necessary orthogonality to $h_{\mathrm{ex}}$, the spin-zero triplets rotate to form equal-spin triplets $[8,9]$. The overall conversion efficiency of spin singlets to equal-spin triplets is then expected to scale with both the amplitude of $h_{\text {ex }}$ and the SOC strength $[8,9]$.

Recent experiments [10-12] have explored the potential role that SOC may play in generating the spin-triplet pair correlations in SC/FM proximity-coupled systems. In particular,

\footnotetext{
*Authors to whom correspondence should be addressed: cc538@cam.ac.uk

†mb52@cam.ac.uk
}

our recent ferromagnetic resonance (FMR) study [10] showed that when strong SOC spin sinks (Ta, W, Pt) are added on either side of $\mathrm{Nb} / \mathrm{Ni}_{80} \mathrm{Fe}_{20} / \mathrm{Nb}$ samples, spin pumping $[13,14]$ from the precessing $\mathrm{Ni}_{80} \mathrm{Fe}_{20}$ into the $\mathrm{Nb}$ can be substantially larger deep in the superconducting state compared with the normal state. This is the opposite behavior to what is expected for the spin-singlet superconductivity [15-17], and is attributed to the flow of spin angular momentum through the proximity-induced equal-spin triplet states by SOC, either at the $\mathrm{Ni}_{80} \mathrm{Fe}_{20} / \mathrm{Nb}$ interface $[8,9]$ or possibly at the $\mathrm{Nb} / \mathrm{Pt}$ interface acting in combination with the Landau Fermi-liquid effect [18].

To better understand the mechanisms contributing to enhanced spin pumping in the superconducting state, we have conducted a series of experiments on $\mathrm{Fe} / \mathrm{Pt} / \mathrm{Nb} / \mathrm{Ni}_{80} \mathrm{Fe}_{20} / \mathrm{Nb} / \mathrm{Pt} / \mathrm{Fe}$ structures. Here the ferromagnetic $\mathrm{Fe}$ layers serve as an internal source of $h_{\mathrm{ex}}$ to the neighboring Pt spin sink [Fig. 1(a)], creating spontaneous spin splitting, which is known to extend to $\mathrm{Pt}$ thicknesses of several nanometers [19]. By comparison with FMR results on $\mathrm{Pt} / \mathrm{Nb} / \mathrm{Ni}_{80} \mathrm{Fe}_{20} / \mathrm{Nb} / \mathrm{Pt}$ control structures without the Fe layers, approximately one order of magnitude enhancement is achieved for certain Pt thicknesses $t_{\mathrm{Pt}}$, but this enhancement disappears for large and small $t_{\mathrm{Pt}}$, demonstrating the requirement for both SOC and the exchange field in generating substantial superconducting spin currents.

We measured the $t_{\mathrm{Pt}}$ dependence of the magnetization $M$ [Fig. 1(b)] and the superconducting transition $T_{\mathrm{c}}$ [Fig. 1(c)] for the two series of samples, with and without the Fe layers. The total $M$ is clearly enhanced by the addition of the $\mathrm{Fe}$ layers and it is independent of $t_{\mathrm{Pt}}$, implying that no significant intermixing/interdiffusion occurs at the $\mathrm{Pt} / \mathrm{Fe}$ interfaces in any of the samples studied. A noteworthy aspect as a function of 
(a)

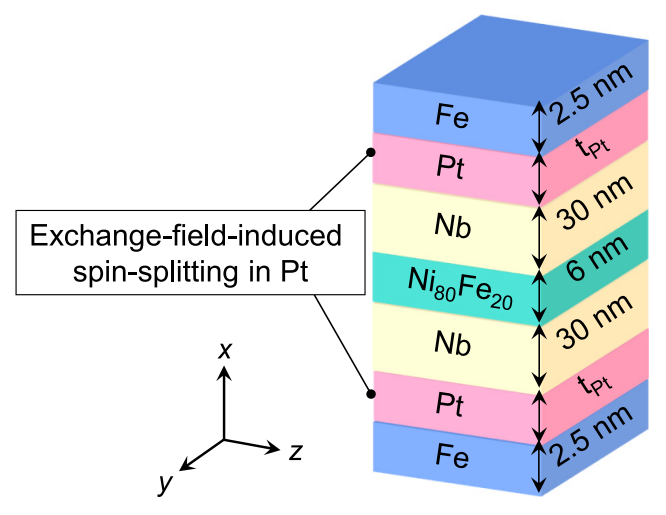

(b)

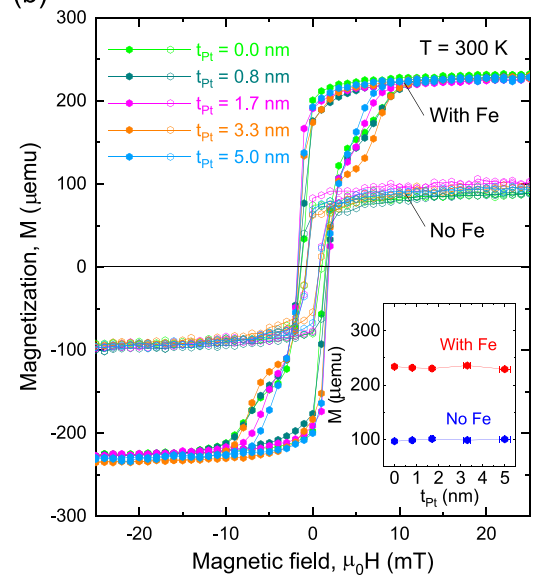

(c)

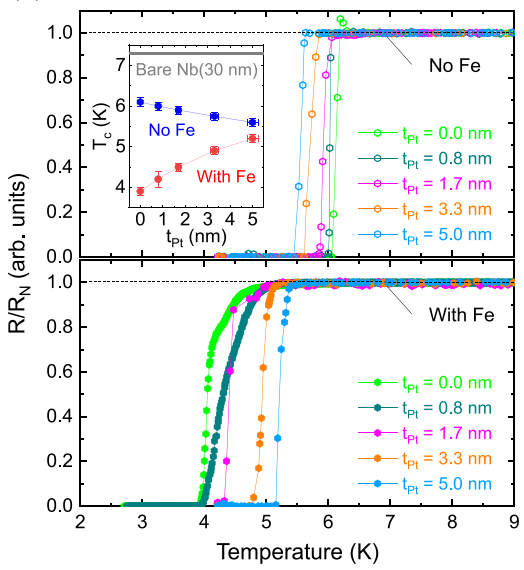

FIG. 1. Structural, magnetic properties, and induced exchange field in $\mathrm{Fe} / \mathrm{Pt} / \mathrm{Nb} / \mathrm{Ni}_{80} \mathrm{Fe}{ }_{20} / \mathrm{Nb} / \mathrm{Pt} / \mathrm{Fe}$ structures. (a) Schematic of the $\mathrm{Fe}(2.5 \mathrm{~nm}) / \mathrm{Pt}\left(t_{\mathrm{Pt}}\right) / \mathrm{Nb}(30 \mathrm{~nm}) / \mathrm{Ni}_{80} \mathrm{Fe}_{20}(6 \mathrm{~nm}) / \mathrm{Nb}(30 \mathrm{~nm}) / \mathrm{Pt}\left(t_{\mathrm{Pt}}\right) / \mathrm{Fe}(2.5 \mathrm{~nm})$ samples with different Pt thicknesses $t_{\mathrm{Pt}}$ and a Cartesian coordinate system used in the present study. (b) In-plane magnetization $M$ curves of the two series of samples with and without the Fe layers. The inset summarizes the $t_{\mathrm{Pt}}$ dependence of total $M$ of the samples. (c) Normalized resistance $R / R_{\mathrm{N}}$ vs temperature $T$ plots for the two series of samples with and without the Fe layers. The inset summarizes the $t_{\mathrm{Pt}}$ dependence of the superconducting transition temperature $T_{\mathrm{c}}$ of the samples; for comparison, $T_{\mathrm{c}}$ of a bare $\mathrm{Nb}(30 \mathrm{~nm})$ film is also shown.

$t_{\mathrm{Pt}}$ is found in the $T_{\mathrm{c}}$ curves: $T_{\mathrm{c}}$ is strongly suppressed by the presence of the Fe layers (about $2 \mathrm{~K}$ for $t_{\mathrm{Pt}}=0 \mathrm{~nm}$ ) and the $T_{\mathrm{c}}$ difference becomes smaller as $t_{\mathrm{Pt}}$ increases. This proves that the added Fe layers affect the (singlet) superconducting properties of the $\mathrm{Nb}$ layer via the inverse proximity effect: that is, the propagation of Fe-induced exchange (spin) splitting transmitted through the $\mathrm{Pt}$ spacer layer to the $\mathrm{Nb} / \mathrm{Pt}$ interface $[20,21]$.

To investigate how the Fe-induced $h_{\mathrm{ex}}$ influences spin transport, we measured the temperature $(T)$ evolution of the FMR spectra, for instance, the FMR linewidth $\left(\mu_{0} \Delta H\right)$ (directly linked to the Gilbert damping $\alpha$ and a measure of the net spin current out of the $\mathrm{Ni}_{80} \mathrm{Fe}_{20}$ ) and the resonance field (associated with the saturation magnetization $\mu_{0} M_{\mathrm{s}}$ ) $[10,13,14]$. Note that the zero-frequency line broadening $\mu_{0} \Delta H_{0}$ in our system has been found to be less than $|0.5 \mathrm{mT}|$, which is negligibly small for the high-frequency regime $(\geqslant 10 \mathrm{GHz})$ [10]. Figure 2(a) shows $\mu_{0} \Delta H$ versus the normalized temperature $T / T_{\mathrm{c}}$ for $\mathrm{Pt} / \mathrm{Nb} / \mathrm{Ni}_{80} \mathrm{Fe}_{20} / \mathrm{Nb} / \mathrm{Pt}$ control structures with different $t_{\mathrm{Pt}}$, taken at a fixed microwave frequency $f=$ $20 \mathrm{GHz}$. We note that the role of the Pt layers in our system is twofold. One is to proximity-induce equal-spin triplet states in the $\mathrm{Nb}$ layers via SOC in combination with $h_{\mathrm{ex}}[8,9]$; the other is to provide a dump for spin angular momentum emitted from the middle $\mathrm{Ni}_{80} \mathrm{Fe}_{20}$ layer through the induced triplet states (of the $\mathrm{Nb}$ ) - a consequence of the very short spin-flip length in $\mathrm{Pt}$ [13]. The resulting flow/transfer of spin angular momentum through proximity-induced (equal-spin) triplet states into singlet SCs, namely, superconducting spin currents, can then be probed by FMR linewidth broadening or Gilbert damping increase of the middle $\mathrm{Ni}_{80} \mathrm{Fe}_{20}[10,13]$. In the normal state $\left(T / T_{\mathrm{c}}>1\right), \mu_{0} \Delta H$ is almost $T$ independent for all $t_{\mathrm{Pt}}$, but increases with increasing $t_{\mathrm{Pt}}$ as the Pt becomes a more effective sink for spin current. Upon entering the superconducting state $\left(T / T_{\mathrm{c}}<1\right)$, a significant $t_{\mathrm{Pt}}$-dependent evolution of $\mu_{0} \Delta H\left(T / T_{\mathrm{c}}\right)$ takes place: a gradual transition from the narrowing to the broadening of $\mu_{0} \Delta H$ with the increase of $t_{\mathrm{Pt}}$. This is basically consistent with our previous findings [10], which can be explained by the enhanced spin transfer via induced (equal-spin) triplet states in the $\mathrm{Nb}$ via SOC $[8,9,18]$ associated with the presence of the Pt $(5 \mathrm{~nm})$, contrasting with the blocking of spin transport in the samples with small or zero $t_{\mathrm{Pt}}$ overwhelmed by the singlet superconductivity.

For these Fe-absent control samples, the amplitude of the spin transfer in the superconducting state as measured by $\mu_{0} \Delta H$ is positively correlated with $t_{\mathrm{Pt}}$. As in the normal state, the effective Pt spin conductance which controls the amount of spin current outflowing [14] from the precessing $\mathrm{Ni}_{80} \mathrm{Fe}_{20}$ diminishes with reducing $t_{\mathrm{Pt}}$; in addition, the interfacial $\mathrm{Nb} / \mathrm{Pt}$ SOC which generates triplet spin supercurrents $[10,18]$ should also quickly decrease as $t_{\mathrm{Pt}}$ goes to zero.

Figure 2(b) displays $\mu_{0} \Delta H\left(T / T_{\mathrm{c}}\right)$ for $\mathrm{Fe} / \mathrm{Pt} / \mathrm{Nb} /$ $\mathrm{Ni}_{80} \mathrm{Fe}_{20} / \mathrm{Nb} / \mathrm{Pt} / \mathrm{Fe}$ structures with several $t_{\mathrm{Pt}}$. In the normal state, the behavior is very similar to that of the control samples shown in Fig. 2(a), demonstrating that the addition of the $\mathrm{Fe}$ does not enhance the normal spin current. A distinctively different behavior of $\mu_{0} \Delta H$ as a function of $t_{\mathrm{Pt}}$ appears in the superconducting state when the Fe layers are present-Fig. 2(b) shows that as $t_{\mathrm{Pt}}$ increases, the low $T$ suppression of FMR damping for the zero $t_{\mathrm{Pt}}$ sample changes to a large damping enhancement at a thinner $t_{\mathrm{Pt}}$, with the largest enhancement at the intermediate $t_{\mathrm{Pt}}$ of $1.7 \mathrm{~nm}$. This is followed by a slow decrease in damping with $\mu_{0} \Delta H$ enhancement for the thickest Pt layer $(5 \mathrm{~nm})$, similar to the sample without the Fe layers.

To characterize the specific difference in $t_{\mathrm{Pt}}$ dependence between the two series of the samples with [Fig. 2(d)] and without [Fig. 2(c)] the Fe layers, we plotted $\mu_{0} \Delta H\left(t_{\mathrm{Pt}}\right)$ for different (constant) $T$, ranging from 80 to $2 \mathrm{~K}$. For the normal state $\left(T / T_{\mathrm{c}}>1\right)$, regardless of the presence of the Fe, $\mu_{0} \Delta H$ increases in an exponential fashion as a function of $t_{\mathrm{Pt}}$, as expected for diffusive spin transport with the increased $\mathrm{Pt}$ spin conductance $[13,14]$. This normal-state behavior can be 
(a) $\quad \mathrm{T}_{\mathrm{c}}=6.1,6.0,5.9,5.75,5.6 \mathrm{~K}$

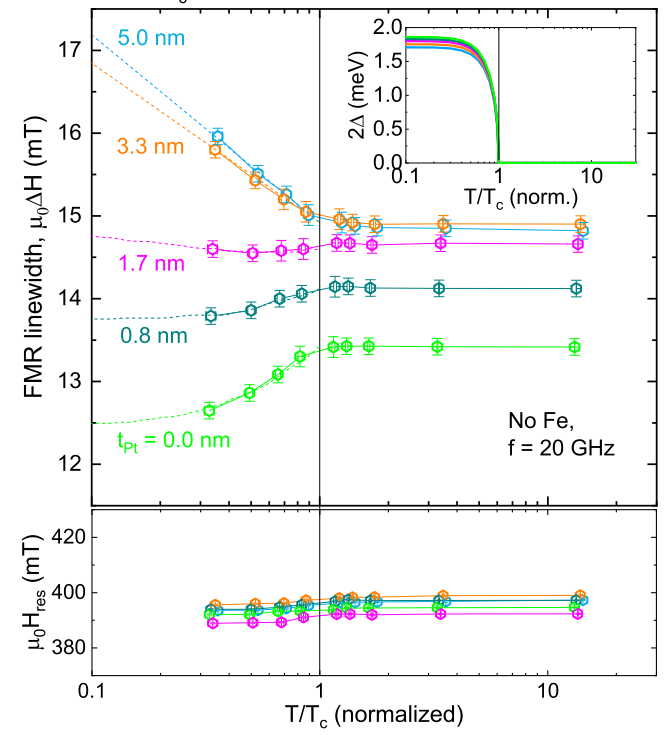

(c)

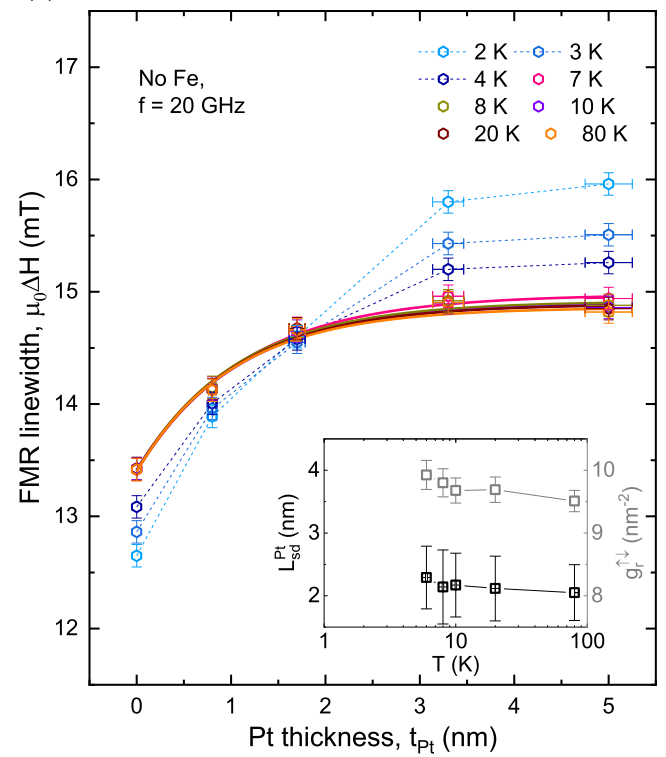

(b) $\quad \mathrm{T}_{\mathrm{c}}=3.9,4.2,4.5,4.9,5.2 \mathrm{~K}$

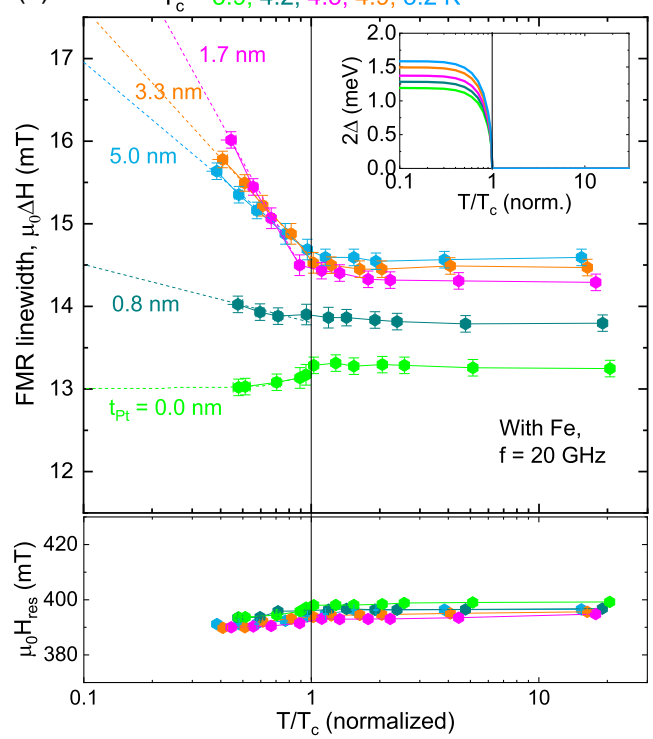

(d)

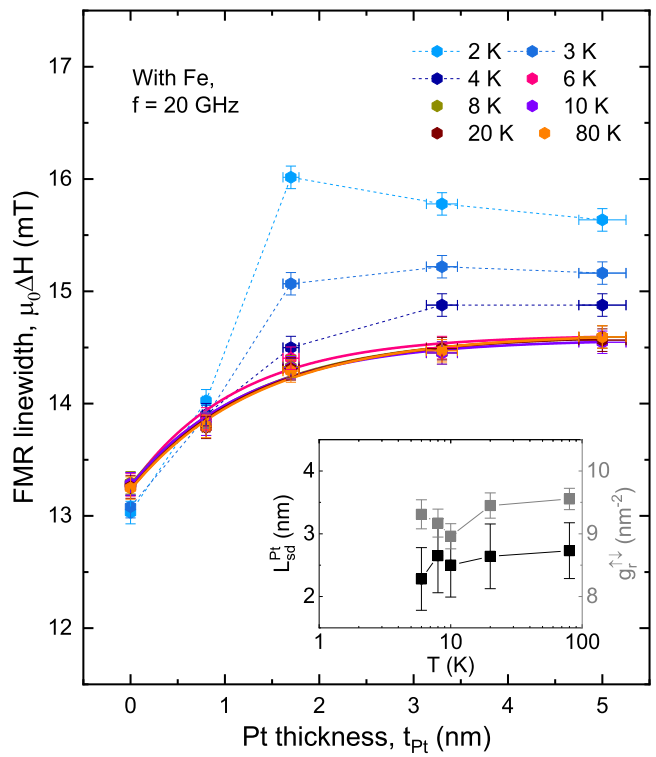

FIG. 2. Characterization of exchange-field effect on spin transport in the superconducting state. (a) Normalized temperature $T / T_{\mathrm{c}}$ dependence of the FMR linewidth $\mu_{0} \Delta H$ (top) and the resonance magnetic field $\mu_{0} H_{\mathrm{res}}$ (bottom) for $\mathrm{Pt}\left(t_{\mathrm{Pt}}\right) / \mathrm{Nb}(30 \mathrm{~nm}) / \mathrm{Ni}_{80} \mathrm{Fe}_{20}(6 \mathrm{~nm}) / \mathrm{Nb}(30 \mathrm{~nm}) / \mathrm{Pt}\left(t_{\mathrm{Pt}}\right)$ control samples with various $\mathrm{Pt}$ thicknesses $t_{\mathrm{Pt}}$. The dashed lines in the top panel are given as guides to the eye. The inset shows the calculated superconducting energy gap $2 \Delta\left(t_{\mathrm{Pt}}\right)$ from the measured $T_{\mathrm{c}}\left(t_{\mathrm{Pt}}\right)$ [Fig. 1(c)] as a function of $T / T_{\mathrm{c}}$. This provides information about how much the added Fe layers further suppress $2 \Delta\left(t_{\mathrm{Pt}}\right)$ via the inverse proximity effect $[20,21]$ in addition to the conventional (singlet) superconducting proximity effect. (b) Data equivalent to (a) but for $\mathrm{Fe}(2.5 \mathrm{~nm}) / \mathrm{Pt}\left(t_{\mathrm{Pt}}\right) / \mathrm{Nb}(30 \mathrm{~nm}) / \mathrm{Ni}_{80} \mathrm{Fe}_{20}(6 \mathrm{~nm}) / \mathrm{Nb}(30 \mathrm{~nm}) / \mathrm{Pt}\left(t_{\mathrm{Pt}}\right) / \mathrm{Fe}(2.5 \mathrm{~nm})$ samples. (c) FMR linewidth $\mu_{0} \Delta H$ as a function of $t_{\mathrm{Pt}}$ of the $\mathrm{Pt} / \mathrm{Nb} / \mathrm{Ni}_{80} \mathrm{Fe}_{20} / \mathrm{Nb} / \mathrm{Pt}$ control samples at various $T$. The solid lines are fits to estimate the effective values of spin mixing conductance at the $\mathrm{Ni}_{80} \mathrm{Fe}_{20} / \mathrm{Nb}$ interface and spin diffusion length of the Pt using the spin pumping model $[13,14]$. The inset shows data and fits for the normal state. (d) Data equivalent to (c) but for the $\mathrm{Fe} / \mathrm{Pt} / \mathrm{Nb} / \mathrm{Ni}_{80} \mathrm{Fe}_{20} / \mathrm{Nb} / \mathrm{Pt} / \mathrm{Fe}$ samples.

quantified using the spin pumping theory $[13,14]$ :

$$
\alpha_{\mathrm{sp}}\left(t_{\mathrm{SC}}, t_{\mathrm{NM}}\right)=2\left(\frac{g_{L} \mu_{B} g_{r}^{\uparrow \downarrow}}{4 \pi M_{s} t_{\mathrm{FM}}}\right)\left[1+g_{r}^{\uparrow \downarrow} \mathcal{R}_{\mathrm{SC}}\left(\frac{1+g^{*} \mathcal{R}_{\mathrm{SC}} \tanh \left(\frac{t_{\mathrm{SC}}}{l_{\mathrm{sd}}^{\mathrm{SC}}}\right)}{\tanh \left(\frac{t_{\mathrm{SC}}}{l_{\mathrm{sd}}^{\mathrm{SC}}}\right)+g^{*} \mathcal{R}_{\mathrm{SC}}}\right)\right]^{-1}, \quad g^{*}\left(t_{\mathrm{NM}}\right)=g\left[1+\frac{g \mathcal{R}_{\mathrm{NM}}}{\tanh \left(\frac{t_{\mathrm{NM}}}{l_{\mathrm{sd}}^{\mathrm{NM}}}\right)}\right]^{-1},
$$

where $g_{L}$ is the Landé $g$ factor, $\mu_{B}$ is the Bohr magneton, and $\hbar$ is Plank's constant divided by $2 \pi . g_{r}^{\uparrow \downarrow}$ is the (effective) spin mixing conductance of the $\mathrm{Ni}_{80} \mathrm{Fe}_{20} / \mathrm{Nb}$ interface and $g$ is the (effective) spin transfer conductance of the $\mathrm{Nb} / \mathrm{Pt}$ 
interface $\left(\sim 35 \mathrm{~nm}^{-2}\right)[13,22] . \mathcal{R}_{\mathrm{SC}(\mathrm{NM})} \equiv \rho_{\mathrm{SC}} l_{\mathrm{sd}}^{\mathrm{SC}(\mathrm{NM})} e^{2} / 2 \pi \hbar$ is the spin resistance of the $\mathrm{Nb}(\mathrm{Pt})$ layer, where $\rho_{\mathrm{SC}}$ is the resistivity of the $\mathrm{Nb}[10], l_{\mathrm{sd}}^{\mathrm{SC}(\mathrm{NM})}$ is the spin diffusion length of the $\mathrm{Nb}(\mathrm{Pt})$, and $e$ is the electron charge. $t_{\mathrm{FM}}$ is the $\mathrm{Ni}_{80} \mathrm{Fe}_{20}$ thickness and $M_{s}$ is its saturation magnetization. Note that the prefactor 2 takes into account the spin pumping through double $\mathrm{Ni}_{80} \mathrm{Fe}_{20} / \mathrm{Nb}$ interfaces [13]. We assumed in Eq. (1) that the addition of 2.5-nm-thick Fe layers does not much affect the overall spin pumping effect since its spin conductance $\left(<3 \mathrm{~nm}^{-2}\right)$ is small relative to other layers [23] direct evidence for this is the very similar FMR linewidths for the $t_{\mathrm{Pt}}=0$ samples with and without Fe shown in Fig. 2 . The similar values of $g_{r}^{\uparrow \downarrow}\left(9-10 \mathrm{~nm}^{-2}\right)$ and $l_{\mathrm{sd}}^{\mathrm{NM}}(2-3 \mathrm{~nm})$ are extracted from fitting Eq. (1) to the data of Figs. 2(c) and 2(d), implying comparable spin injection/transport properties of both samples in the normal state. The estimated $l_{\mathrm{sd}}^{\mathrm{NM}}(2-3 \mathrm{~nm})$ is consistent with that obtained from the spin pumping and inverse spin Hall effect in FM metal/Cu/Pt structures where spin-memory loss at interfaces (i.e., interface spin-flip scattering) can be neglected [22,24].

However, for the superconducting state $\left(T / T_{\mathrm{c}}<1\right)$, $\mu_{0} \Delta H\left(t_{\mathrm{Pt}}\right)$ is affected strongly by the presence of the $\mathrm{Fe}$ layers. From a comparison of Figs. 2(c) and 2(d), we can see that there is a clear rise in the $\mu_{0} \Delta H$ enhancement for the $t_{\mathrm{Pt}}=1.7 \mathrm{~nm}$ sample with the Fe layers. Note also that the superconducting state $\mu_{0} \Delta H\left(t_{\mathrm{Pt}}\right)$ deviates from the exponential fashion for both sample sets [Figs. 2(c) and 2(d)] and so it cannot be fitted by Eq. (1). All these results point to a fundamentally different spin transfer mechanism at play deep in the superconducting state when coupled to either Pt or $\mathrm{Pt} / \mathrm{Fe}$ spin sink.

We show below that this spin transfer phenomenon is consistent with a proximity-induced equal-spin triplet pairing generated by SOC $[8,9,18]$ and enhanced by the Fe-induced exchange (spin) splitting in the Pt.

A quantitative analysis of the effect of the Fe-induced $h_{\mathrm{ex}}$ on the superconducting spin transport is available in our present study by comparing the $\mu_{0} \Delta H$ difference across $T_{\mathrm{c}}$, defined as $\Delta\left[\mu_{0} \Delta H\right]=\mu_{0} \Delta H\left(0.5 T_{\mathrm{c}}\right)-\mu_{0} \Delta H\left(1.5 T_{\mathrm{c}}\right)$, with and without the Fe layers as a function of $t_{\mathrm{Pt}}$ [Fig. 3(a)]. In the absence of the Fe layers, $\Delta\left[\mu_{0} \Delta H\right]$ monotonically rises with increasing $t_{\mathrm{Pt}}$ and shifts from negative (representing the blocking effect of dominant singlet superconductivity) to positive (indicating enhanced spin transport mediated by triplet pairing). However, when the Fe layers are present, this enhancement becomes more pronounced up to $t_{\mathrm{Pt}}=1.7 \mathrm{~nm}$ followed by a fall to the almost same value for larger thicknesses.

There are several competing effects which lead to this maximum at intermediate thicknesses for the Fe-added samples. First, the interfacial $\mathrm{Nb} / \mathrm{Pt} /(\mathrm{Fe}) \mathrm{SOC}$ which appears to be required for triplet spin supercurrent generation [10] should vanish for both sample sets as $t_{\mathrm{Pt}}$ goes to zero-in this case, there is no triplet pairing and the spin transport via singlet superconducting states should be lower than in the normal state-thus the $t_{\mathrm{Pt}}=0$ data is similar and negative for both sample sets. Second, because the spin conductance of the $\mathrm{Fe}$ layers is very small (relative to the Pt layers) [23], the overall $\mathrm{Pt} /(\mathrm{Fe})$ spin conductance should be reduced with decreasing $t_{\mathrm{Pt}}$ so that for small $t_{\mathrm{Pt}}$, even if triplet pairs are generated, (a)

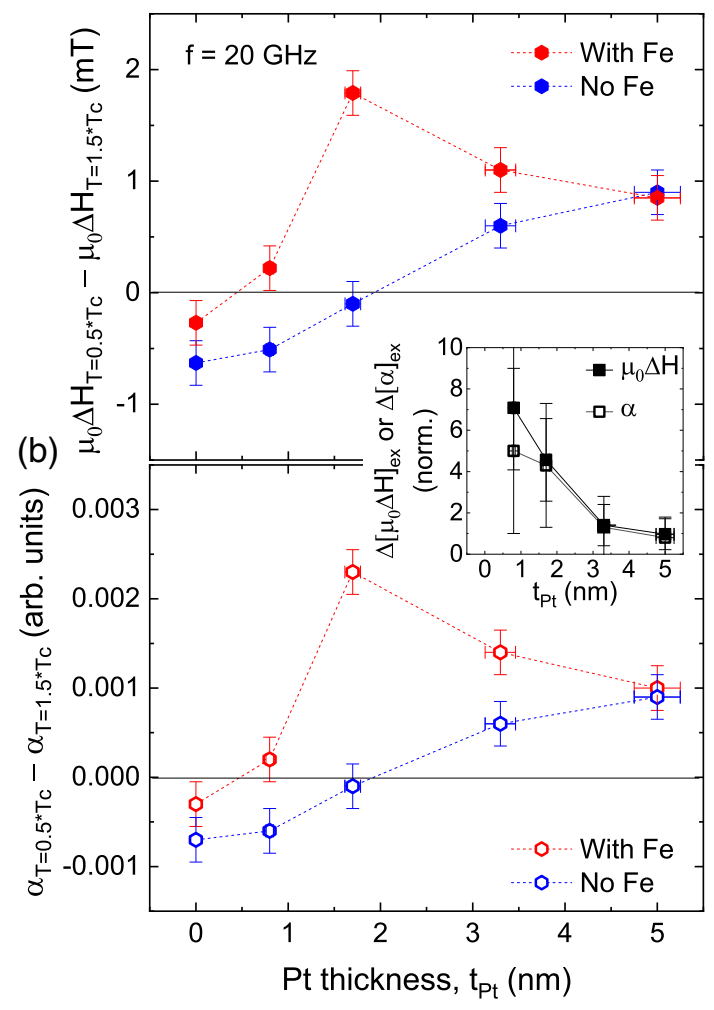

FIG. 3. Exchange-field-enhanced spin transport in the superconducting state. (a) Pt thickness $t_{\mathrm{Pt}}$ dependence of the FMR linewidth $\mu_{0} \Delta H$ difference across $T_{\mathrm{c}}$, defined as $\Delta\left[\mu_{0} \Delta H\right]=$ $\mu_{0} \Delta H\left(0.5 \cdot T_{\mathrm{c}}\right)-\mu_{0} \Delta H\left(1.5 \cdot T_{\mathrm{c}}\right)$, with and without the Fe layers. (b) Data equivalent to (a), but for the Gilbert damping $\alpha[13,14]$. The inset shows the estimated contribution of the Fe-induced exchange field $h_{\mathrm{ex}}$ to the spin transport, denoted as $\Delta\left[\mu_{0} \Delta H\right]_{\mathrm{ex}}$ or $\Delta[\alpha]_{\mathrm{ex}}$, as a function of $t_{\mathrm{Pt}}$.

the absorption of superconducting spin currents by the $\mathrm{Pt}$ is inactive. Note that the net flow of spin angular momentum through the induced triplet states by SOC (which is what is measured by the FMR spectroscopy) predominantly depends on the effective Pt spin conductance, which tends to increase until the Pt thickness becomes comparable to its spin diffusion length [13]. Finally, the exchange field at the $\mathrm{Nb} / \mathrm{Pt}$ interface is known to increase rapidly with decreasing $t_{\mathrm{Pt}}$ in $\mathrm{Pt} / \mathrm{Fe}$ [19] so that if singlet-to-triplet pair conversion is indeed further enhanced by the induced $h_{\mathrm{ex}}$, this effect would decay with increasing $t_{\mathrm{Pt}}$, and for large $t_{\mathrm{Pt}}$, one would expect the data from the two sample sets to become identical as is the case of for the $t_{\mathrm{Pt}}=5 \mathrm{~nm}$ samples.

Taking these effects together, one can see that an intermediate maximum of superconducting spin current might be expected for the samples with $\mathrm{Fe}$ as the rapid increase in the induced $h_{\mathrm{ex}}$ and hence triplet pair density with decreasing $t_{\mathrm{Pt}}$ counteracts the reducing SOC and spin conductance associated with the Pt until the disappearance of the Pt removes the spin sink and SOC from the system at it reverts to singlet behavior.

One can, in principle, isolate the contribution of the Fe-induced $h_{\mathrm{ex}}\left(t_{\mathrm{Pt}}\right)$ from the other effects of changing 
Pt thickness by normalizing the $t_{\mathrm{Pt}}$-dependent enhancement of $\Delta\left[\mu_{0} \Delta H\right]$ with the Fe layers (red symbol in
Fig. 3) to that without the Fe layers (blue symbol) as follows:

$$
\Delta\left[\mu_{0} \Delta H\left(t_{\mathrm{Pt}}\right)\right]_{\mathrm{ex}}=\frac{\left\{\Delta\left[\mu_{0} \Delta H\left(t_{\mathrm{Pt}}\right)\right]-\Delta\left[\mu_{0} \Delta H\left(t_{\mathrm{Pt}}=0\right)\right]\right\}^{\mathrm{w} / \mathrm{Fe}}}{\left\{\Delta\left[\mu_{0} \Delta H\left(t_{\mathrm{Pt}}\right)\right]-\Delta\left[\mu_{0} \Delta H\left(t_{\mathrm{Pt}}=0\right)\right]\right\}^{\mathrm{w} / \mathrm{o} \mathrm{Fe}}} .
$$

The inset of Fig. 3 shows that $\Delta\left[\mu_{0} \Delta H\right]_{\text {ex }}$ goes up rapidly with reducing $t_{\mathrm{Pt}}$, reaching a factor of about 7.5 for $0.8 \mathrm{~nm}$. Essentially, the same behavior was observed in an analysis based on FMR damping $\alpha$ [Fig. 3(b)], extracted from $\mu_{0} \Delta H(f)[10,13,14]$ (see the Supplemental Material [25] for full details).

We have shown that the spin angular momentum transfer into singlet SCs can be further enhanced by one order of magnitude when spontaneous spin splitting in the Pt spin sink is induced by the addition of FM layers. The understanding of SOC generation of superconducting spin currents is still evolving, but the latest theory $[18,36]$ highlights the need for an induced exchange field in the SOC material. For the Fe-absent samples as reported in our previous paper [10], this is indirectly applied by the spin accumulation at the Pt interface, transmitted by the triplet spin current itself, in combination with Landau Fermi-liquid interactions. The key finding of this paper is that superconducting spin pumping can be dramatically enhanced by the influence of the direct exchange field of a coupled ferromagnetic layer on the properties of the Pt layer. This not only provides experimental support for the existing theory of triplet mediated transport $[8,9,18]$, but provides a basis for the development of the comprehensive understanding and optimization of superconducting spin transport.

This work was supported by EPSRC Programme Grant No. EP/N017242/1.
[1] J. Linder and J. W. A. Robinson, Nat. Phys. 11, 307 (2015).

[2] M. Eschrig, Rep. Prog. Phys. 78, 104501 (2015).

[3] N. O. Birge, Philos. Trans. R. Soc. A 376, 20150150 (2018).

[4] M. G. Blamire and J. W. A. Robinson, J Phys: Condens. Matter 26, 453201 (2014).

[5] N. Banerjee, C. B. Smiet, R. G. J. Smits, A. Ozaeta, F. S. Bergeret, M. G. Blamire, and J. W. A. Robinson, Nat. Commun. 5, 4771 (2014).

[6] X. L. Wang, A. Di Bernardo, N. Banerjee, A. Wells, F. S. Bergeret, M. G. Blamire, and J. W. A. Robinson, Phys. Rev. B 89, 140508(R) (2014).

[7] A. Srivastava, L. A. B. Olde Olthof, A. Di Bernardo, S. Komori, M. Amado, C. Palomares-Garcia, M. Alidoust, K. Halterman, M. G. Blamire, and J. W. A. Robinson, Phys. Rev. Appl. 8, 044008 (2017).

[8] F. S. Bergeret and I. V. Tokatly, Phys. Rev. B 89, 134517 (2014).

[9] S. H. Jacobsen, J. A. Ouassou, and J. Linder, Phys. Rev. B 92, 024510 (2015).

[10] K.-R. Jeon et al., Nat. Mater. 17, 499 (2018).

[11] N. Banerjee, J. A. Ouassou, Y. Zhu, N. A. Stelmashenko, J. Linder, and M. G. Blamire, Phys. Rev. B 97, 184521 (2018).

[12] N. Satchell and N. O. Birge, Phys. Rev. B 97, 214509 (2018).

[13] Y. Tserkovnyak, A. Brataas, G. E. W. Bauer, and B. I. Halperin, Rev. Mod. Phys. 77, 1375 (2005).

[14] K. Ando et al., J. Appl. Phys. 109, 103913 (2011).

[15] C. Bell, S. Milikisyants, M. Huber, and J. Aarts, Phys. Rev. Lett. 100, 047002 (2008).

[16] J. P. Morten, A. Brataas, G. E. W. Bauer, W. Belzig, and Y. Tserkovnyak, Eur. Phy. Lett. 84, 57008 (2008).

[17] T. Wakamura, N. Hasegawa, K. Ohnishi, Y. Niimi, and Y. C. Otani, Phys. Rev. Lett. 112, 036602 (2014).

[18] X. Montiel and M. Eschrig, Phys. Rev. B 98, 104513 (2018).

[19] C. Klewe, T. Kuschel, J.-M. Schmalhorst, F. Bertram, O. Kuschel, J. Wollschläger, J. Strempfer, M. Meinert, and G. Reiss, Phys. Rev. B 93, 214440 (2016).
[20] M. Vélez, C. Martínez, A. Cebolladab, F. Brionesb, and J. L. Vicentc, J. Magn. Magn. Mater. 240, 580 (2002).

[21] H. Yamazaki, N. Shannon, and H. Takagi, Phys. Rev. B 81, 094503 (2010).

[22] J. C. Rojas-Sanchez, N. Reyren, P. Laczkowski, W. Savero, J. P. Attane, C. Deranlot, M. Jamet, J. M. George, L. Vila, and H. Jaffres, Phys. Rev. Lett. 112, 106602 (2014).

[23] T. Tanaka, H. Kontani, M. Naito, T. Naito, D. S. Hirashima, K. Yamada, and J. Inoue, Phys. Rev. B 77, 165117 (2008).

[24] M. Caminale, A. Ghosh, S. Auffret, U. Ebels, K. Ollefs, F. Wilhelm, A. Rogalev, and W. E. Bailey, Phys. Rev. B 94, 014414 (2016).

[25] See Supplemental Material at http://link.aps.org/supplemental/ 10.1103/PhysRevB.99.024507 for a detailed analysis of the MW frequency dependence of FMR spectra for the samples with and without the Fe layers at low temperatures, effect of the $\mathrm{Fe}$ thickness on the overall FMR spectra of $\mathrm{Fe} / \mathrm{Pt} / \mathrm{Nb} / \mathrm{Ni}_{80} \mathrm{Fe}_{20} / \mathrm{Nb} / \mathrm{Pt} / \mathrm{Fe}$ samples, and experimental details, which includes Refs. [14,26-35].

[26] Z. Celinski, K. B. Urquhart, and B. Heinrich, J. Magn. Magn. Mater. 166, 6 (1997).

[27] B. Heinrich, Ultrathin Magnetic Structures (Springer, Berlin, 2005), Vol. III.

[28] Y. Tserkovnyak, A. Brataas, and G. E. W. Bauer, Phys. Rev. Lett. 88, 117601 (2002).

[29] C. Kittel, Phys. Rev. 73, 155 (1948).

[30] J. M. Shaw, H. T. Nembach, T. J. Silva, and C. T. Boone, J. Appl. Phys. 114, 243906 (2013).

[31] E. Montoya, P. Omelchenko, C. Coutts, N. R. Lee-Hone, R. Hubner, D. Broun, B. Heinrich, and E. Girt, Phys. Rev. B 94, 054416 (2016)

[32] S. S. Kalarickal, J. Appl. Phys. 99, 093909 (2006).

[33] S. Mizukami, Y. Ando, and T. Miyazaki, Jpn. J. Appl. Phys. 40, 580 (2001). 
[34] P. Omelchenko, E. A. Montoya, C. Coutts, B. Heinrich, and E. Girt, Sci. Rep. 7, 4861 (2018).

[35] A. I. Gubin, K. S. Il'in, S. A. Vitusevich, M. Siegel, and N. Klein, Phys. Rev. B 72, 064503 (2005).

[36] In our theoretical work [18], the equilibrium spin currents have been calculated to demonstrate the long-range triplet correlations, but they are not to be equated with the pumped nonequilibrium spin currents. Although quantitative details and mathematical descriptions need to be fully set out, it is evident that with the presence of equilibrium equal-spin triplet pairs in the entire structure, a new channel is opened for spin currents to be transmitted through a singlet SC to Pt spin sink. 